\title{
Riemann Solvers for some Hyperbolic Problems with a Source Term
}

\author{
Alain Yves LE ROUX
}

Résumé. Dans de nombreux modèles de mécanique des fluides, le terme source correspond à une interaction de l'écoulement avec le milieu environnant : la géométrie ou certaines forces internes telles que la friction, la gravité, la force de Coriolis, etc.. Dans les problèmes d'environnement, cette interaction est souvent un terme dominant qui détermine les équilibres (états au repos). Lors des simulations numériques, il est évidemment très important de reproduire parfaitement ces états d'équilibre et de les maintenir à l'équilibre au cours du temps. Il est surtout important d'éviter que la perte de l'équilibre au niveau numérique vienne déstabiliser et modifier de façon significative la simulation. Dans ce papier, cette question est analysée à partir d'exemples concrets, et des solveurs appropriés, dits solveurs équilibrés, sont proposés

\section{Mots clés.}

Problèmes d'environnement, stabilité de schémas numériques pour les systèmes hyperboliques, solveurs équilibrés, volumes finis (Godunov).

\begin{abstract}
In many problems of fluid dynamics, the source term corresponds to the interaction with the surrounding medium, including the geometry and some internal forces (friction, gravity, Coriolis acceleration, etc..) In environmental problems, this interaction is often a very dominant term which characterizes the well balanced states. In a numerical simulation, it is obviously important to be able to reproduce these equilibria and maintain them for a large number of timesteps, or at least, to avoid a stable equilibrium to be disturbed and destabilized by an unsuitable method. This is the matter discussed in the present paper.
\end{abstract}

Keywords. Problems in environmental science, stability of numerical schemes for hyperbolic systems, well balanced solvers, finite volumes (Godunov)

AMS Subject Classification. 65M05, 65M10

\section{Some examples}

We first consider the following one dimension model,

$$
u_{t}+u(u+a)_{x}=0
$$

Article published by EDP Sciences and available at http://www.edpsciences.org/proc or http://dx.doi.org/10.1051/proc:1999047 
where $a$ is a given function, for example,

$$
a(x)=\frac{a_{0}}{1+K x^{2}},
$$

with given constants $a_{0}>O, K>0$. We start with the following initial data, for a given small $\epsilon>0$,

$$
u(x, 0)=\left\{\begin{array}{cc}
0 & \text { for } x>0, \\
\max \left(0, a_{0}-a(x)-\epsilon\right) & \text { for } x<0,
\end{array}\right.
$$

The solution of this problem is obviously the stationary solution since the flux term $u(u+$ $a)_{x}$ is always zero (either $u=0$ or $u+a=a_{0}-\epsilon=$ constant).

A usual way to solve numerically this problem is to use a splitting technique, that is to cut (1) into two steps which will be run consecutively at each timestep during the computation. These two steps are

$$
\text { Step 1: } \quad u_{t}+\left(\frac{u^{2}}{2}\right)_{x}=0
$$

$$
\text { Step 2: } u_{t}-u a^{\prime}(x)=0 .
$$

Let us consider a point $x<0$, close to 0 . We have $u_{x}<0$ and $u \geq 0$, and from (4) the solution $u$ will increase and move forwards. This can create a value $u_{*}>0$ on the top of $a$ at $x=0$, which will not be corrected by (5) since $a^{\prime}(x)=0$ on the top. We can expect that the solution will still move rightwards and become a shock wave. This shock wave will have the following form

$$
u(x, t)=\left\{\begin{array}{cl}
u_{*}+a_{0}-a(x) & \text { for } 0<x<x(t) \\
0 & \text { for } x>x(t)
\end{array}\right.
$$

where $x=x(t)$ corresponds to the shock path whose equation is given by the Rankine Hugoniot relation

$$
x^{\prime}(t)=\frac{1}{2}\left(u_{*}+a_{0}-a(x)\right),
$$

starting from $x=0$. For a large $x$, we have $x^{\prime}(t) \sim \frac{a_{0}}{2}$ and a jump close to $a_{0}$ (which means a large one). This example shows how a tiny error at the bad place will generate a large error that cannot be controled later. It is a very difficult task to prevent this phenomenon by using a splitted numerical method corresponding to a discretization of (4), (5). A possible way is to use a very small meshsize. One of the aims of this paper is to propose other schemes, which maintain the equilibria. An example of numerical scheme for this model will be presented in Section 3. It is not a splitted method. 


\section{Some physical models}

\subsection{Hydraulics}

We can consider the above function $a(x)$ given in (2) as the profile of a dam with a water reservoir on the $x<0$. We consider the following (so-called) Shallow Water one dimension model

$$
\begin{gathered}
h_{t}+(h u)_{x}=0 \\
(h u)_{t}+\left(h u^{2}\right)_{x}+g h(h+a)_{x}=0,
\end{gathered}
$$

where $h$ is the heigth of water, $u$ the velocity, and $g$ the gravity constant. We can notice that this model is relevant even for deeper flows, as long as the velocity stays homogeneous on the thickness of the water layer; otherwise, this model corresponds to a damped model since the velocity is averaged, which means reduced as seen easily by using the Cauchy Schwartz inequality.

We start with the following initial data

$$
\begin{gathered}
h(x, 0)=\left\{\begin{array}{cc}
0 & \text { for } x>0 \\
\max \left(0, Z_{0}-a(x)\right) & \text { for } x<0,
\end{array}\right. \\
u(x, 0)=0
\end{gathered}
$$

where $Z_{0}$ corresponds to the water level. For $Z_{0}<a_{0}$, we see clearly that the solution is the stationary one since the water will not climb by itself up to the top of the dam.

Now, by using a splitting technique as the following one,

$$
\begin{gathered}
\text { Step 1: }\left\{\begin{array}{c}
h_{t}+(h u)_{x}=0, \\
(h u)_{t}+\left(h u^{2}+g \frac{h^{2}}{2}\right)_{x}=0,
\end{array}\right. \\
\text { Step 2: }\left\{\begin{array}{c}
h_{t}=0 \\
(h u)_{t}=-g h a^{\prime}(x) .
\end{array}\right.
\end{gathered}
$$

We can notice that the first step will move the water rightwards from the left hand side of the dam. If $Z_{0}$ is close to $a_{0}$ (with $Z_{0}<a_{0}$ ), a positive heigth of water will appear on the top of the dam, will provoke a flux forwards, and a flow dropping down the hill. This flow will be a torrential one, with a Froude number $\frac{|u|}{\sqrt{g h}}$ larger that one (this is to be compared to a supersonic flow in hydrodynamics), characterized by a thin layer of water with a large velocity. In such a situation, a numerical scheme can produce a negative value for $h$, and download the computer. Obviously, this cannot be prevented by the second step, since $a^{\prime}(x)$ is zero on the top of the dam, and negative rightwards, which will accelerate the flow. 


\section{$2.2 \quad$ Atmosphere}

We consider the Euler equations with a constant gravity for a one (vertical) dimension. The ground corresponds to $x=0$. The model reads

$$
\begin{gathered}
\rho_{t}+(\rho u)_{x}=0 \\
(\rho u)_{t}+\left(\rho u^{2}+p\right)_{x}=-\rho g, \\
(\rho e)_{t}+((\rho e+p) u)_{x}=-\rho g u,
\end{gathered}
$$

where $\rho$ is the density, $u$ is the velocity, $g$ the gravity constant, $p$ the pressure and $e$ the total energy. The two last parameters $p$ and $e$ are linked by the internal energy $I$ through the formulae

$$
e=I+\frac{u^{2}}{2} \quad, \quad p=(\gamma-1) \rho I,
$$

where $\gamma$ is the adiabatic constant of the air. From (8), (9) and (10), we can reduce (10) to the following equation on the pressure

$$
p_{t}+u p_{x}+\gamma p u_{x}=0
$$

where no source term is present. This shows that the only non homogeneous equation in the system is (9).

We consider now an equilibrium at rest, which means that $u=0$ and all the time derivatives are zero. The equations (8), (10) or (12) are reduced to "zero $=$ zero", and (9) is reduced to the single equation

$$
(\gamma-1)(\rho I)_{x}=-\rho g \quad,
$$

which involves two parameters. We can construct an infinity of solutions. Among them are these two particular ones, for a given state $\left(\rho_{0}, I_{0}\right)$ at the ground level:

$$
I=I_{0}, \quad \rho=\rho e^{-\frac{g x}{(\gamma-1) I_{0}}},
$$

and

$$
I=I_{0}-\frac{g x}{\gamma-1}, \quad \rho=\rho_{0}
$$

The first one corresponds to a strong tornado since the larger part of the air will be pulled up. The second one will produce a negative internal energy at the level $x=(\gamma-1) \frac{I_{0}}{g}$ which has no physical meaning. As a matter of fact, an admissible stationary solution will correspond to experimental measurements and it is really difficult to preserve such an equilibrium numerically. A splitting technique will simulate a catastrophic behaviour of the atmosphere, far stronger than any phenomenon we use to modelize in environmental science. A suitable method is presented in the next section. This method does not correspond to a new scheme, but to a new formulation of the model, which becomes a homogeneous one .

ESA IM: Proc., Vol. 6, Septembre 1998, 75-90 


\section{A well balanced method for the Atmosphere model}

We consider the model of atmosphere described in the previous section, that is (8), (9) and (10) or (12). We recall that $g$ is a constant. The transport equation (8) expresses that the vector $(\rho u, \rho)$ is divergence free in the $(x, t)$ set. Hence, there exists a potential denoted by $\varphi=\varphi(x, t)$ such that

$$
\varphi_{x}=\rho, \quad \varphi_{t}=-\rho u,
$$

which leads to the equation

$$
\varphi_{t}+u \varphi_{x}=0
$$

We set

$$
q=g \varphi,
$$

and by using (8), we get that $\rho q$ satisfies a transport equation of velocity $u$. That way, we have got a new formulation of the model,

$$
\begin{gathered}
(\rho q)_{t}+(\rho q u)_{x}=0 \\
\rho_{t}+(\rho u)_{x}=0 \\
(\rho u)_{t}+\left(\rho u^{2}+p+q\right)_{x}=0 \\
(\rho e-q)_{t}+((\rho u+p) u)_{x}=0 .
\end{gathered}
$$

which is a homogeneous system of conservation laws. It is still an hyperbolic problem whose eigenvalues of the flux matrix are

$$
\lambda=u-c, \lambda=u \text { (double) }, \lambda=u+c, \text { with } c=\sqrt{\frac{\gamma p}{\rho}} .
$$

Moreover, the eigenspace associated with the double eigenvalue $\lambda=u$ has two dimensions, so that the system has the same properties as a strictly hyperbolic one. We find again the same Riemann invariant as for the homogenous version of (8), (9) and (10) and the same shock conditions (derived from the Rankine Hugoniot relations) for the three parameters $\rho, u$ and $p$. On a shock wave we get that $q$ is continuous (no jump). The only difference comes from the contact discontinuities, where the continuous variables are now the velocity $u$ and the total pressure $p+q$, instead of $u$ and $p$ in the previous model. This allows to relax the pressure and help the preservation of the equilibria.

We can now solve this system by using any classical scheme. For exemple the Godunov method works very well and preserve the equilibria even when starting from experimental measured data. More, the numerical tests using the Godunov scheme show that the stability of an initial equilibrium will be recovered after a strong local perturbation ( [3], [4]). The only modifications in the Godunov scheme are the following:

- the Riemann solver is modified by including the balance $p+q=$ constant on the contact discontinuities,

- the source term is taken in account together with the flux term, since it becomes a flux term itself in the new model.

And we add this essential remark: the CFL condition is not perturbed. This is another very important advantage on the splitting technique. 


\section{A well balanced scheme for the scalar model}

We try to adapt the previous idea for the scalar equation

$$
u_{t}+f(u)_{x}=g(u) a(x)
$$

We suppose for convenience that $f, g \in C^{1}(\mathbb{R}), f^{\prime}>0$ and that $a$ is a given function.

A first naive idea is to introduce a function $q$ such that $q_{x}=-g(u) a(x)$, in order to write (16) under the form

$$
u_{t}+(f(u)+q)_{x}=0 .
$$

However, we get no homogeneous equation for $q$ in the general case, and this idea is to be rejected.

Another idea is to introduce a primitive function $A(x)$ of $a(x)$, and give (16) the form

$$
u_{t}+g(u)\left(\frac{f^{\prime}(u)}{g(u)} u_{x}+A^{\prime}(x)\right)=0
$$

which is

$$
u_{t}+g(u) w_{x}=0
$$

by writing successively

$$
\varphi^{\prime}(u)=\frac{f^{\prime}(u)}{g(u)} \quad, \text { and } w=\varphi(u)+A(x) .
$$

We get

$$
w_{t}=\varphi^{\prime}(u) u_{t}=-\frac{f^{\prime}(u)}{g(u)} g(u) w_{x}
$$

that is

$$
w_{t}+f^{\prime}(u) w_{x}=0 .
$$

Now we have a homogenous system, made of (17) and (19) which presents however some drawbacks: it is not a conservative one and a new eigen value $\lambda=0$ has been created. On the other hand, we get from (19) that $w$ has the behaviour of a Riemann invariant along the characteristics of velocity $f^{\prime}(u)$. We shall use it to solve the Riemann problem, noticing that a wide class of function $A(x)$ may be used, including the class of constant piecewise functions. In the construction of the method, we shall approach the function $A(x)$ by a constant piecewise function which is constant on each cell, and use (19) to build the Riemann solver.

Let $\Delta x$ be the meshsize and $\Delta t$ the timestep. We set $x_{i}=i \Delta x$ for $i \in \mathbb{Z}$, and $\left.M_{i}=\right] x_{i}, x_{i+1}\left[\right.$. The approximate solution at time $t_{n}=n \Delta t$ on the cell $M_{i}$ will be denoted $u_{i}^{n}$. We build a constant piecewise function $A_{\Delta x}$ defined by

$$
A_{\Delta x}(x)=A_{i}=\int_{0}^{x_{i}} a(\xi) d \xi \text { for } x \in M_{i}
$$

ESA IM: Proc., Vol. 6, SePtembre 1998, 75-90 
and set, for each cell $M_{i}$

$$
w_{i}^{n}=\varphi\left(u_{i}^{n}\right)+A_{i} .
$$

By using (19), one gets that $w$ is constant across each interface $x=x_{i}$, and travelling forwards along the characteristic $x^{\prime}(t)=f^{\prime}(u)$ with $f^{\prime}(u)>0$. The Riemann problem near the interface $x=x_{i}$ is made of the equation (19) and the starting data

$$
w\left(x, t_{n}\right)=\left\{\begin{array}{l}
w_{i}^{n} \text { for } x>x_{i} \\
w_{i-1}^{n} \text { for } x<x_{i}
\end{array}\right.
$$

We denote by $w_{i}^{*}$ (resp. $u_{i}^{*}$ ) the trace of $w$ (resp. $u$ ) along the right side of the interface $x=x_{i}$. We have $w_{i}^{*}=w_{i-1}^{n}$, which leads to

$$
\varphi\left(u_{i}^{*}\right)=\varphi\left(u_{i-1}^{n}\right)-\left(A_{i}-A_{i-1}\right) .
$$

Since $\varphi$ may be discontinous at the roots of $g$ and continous in the inside of the intervals limited by these roots, we take of course $u_{i}^{*}$ in the interval where $u_{i-1}^{n}$ is lying.

The Godunov scheme, which is here close to the decentered scheme, reads

$$
u_{i}^{n+1}=u_{i}^{n}-r\left(f\left(u_{i}^{*}\right)-f\left(u_{i-1}^{n}\right)\right),
$$

where $r=\frac{\Delta t}{\Delta x}$. We get it immediately by integrating

$$
u_{t}+f(u)_{x}=g(u) A_{\Delta x}^{\prime}(x)
$$

on the set $\left.M_{i} \times\right] t_{n}, t_{n+1}\left[\right.$ and noticing that $A_{\Delta x}^{\prime}$ is zero in the inside of the cell $M_{i}$.

The numerical method is made of (22) and (23) and works under the usual CFL condition for the decentered scheme, that is

$$
r \max f^{\prime}(u) \leq 1 .
$$

One can prove $L^{\infty}$ and $B V$ estimates, and the convergence towards the entropy condition (see $[6],[7],[8]$ ).

This method works obviously in the case $a(x)=1$ (and a given $g(u)$ ), and we notice that the constant $a=1$ is approached by the Dirac comb $\frac{d}{d x} E\left(\frac{x}{\Delta x}\right) \Delta x$ where $E$ states for the integer part. It works obviously in the case $g(u)=1, a=a(x)$ too.

This method has been tested on the example (1), (2), (3) for a small $\epsilon$, and the initial equilibrium was perfectly respected, independently on $\epsilon$. It was not the case for the usual decentered scheme coupled with a splitting technique with the same meshsize. By using a very small meshsize connected with the size of $\epsilon$, we can however expect this splitting method to work, for there is a well known result of convergence. 


\section{The Shallow Water model}

This section is based on the results obtained in [5].

We consider a water flow over either a river bed, or the surrounding ground when flooding, or the bottom of the ocean for a coastal flow. We first consider a one dimension model, and denote by $a=a(x)$ the bottom elevation, by $h$ the heigth of water $(h=0$ corresponds to a dry ground), by $g$ the gravity constant and by $u$ the water velocity when $h>0$. The model is the following

$$
\begin{gathered}
h_{t}+(h u)_{x}=0 \\
(h u)_{t}+\left(h u^{2}\right)_{x}+g h(h+a)_{x}=0
\end{gathered}
$$

which is known as the Shallow Water model, thought it still works for deep water too, in many cases. This model can be built from the uncompressible Euler equations by integrating over the thickness of the water layer. We take the average velocity

$$
u(x, t)=\frac{1}{h} \int_{a}^{a+h} \tilde{u}(x, z, t) d z
$$

where $\tilde{u}$ is the velocity in the Euler model. The equation (25) is derived immediately from the uncompressibility hypothesis in the Euler model, and the equation (26) is obtained by using the hydrostatic pressure, that is

$$
\frac{\partial p}{\partial z}=-g
$$

and the averaged velocity given by $(27)$ instead of $\tilde{u}$. Note that this last approximation can be seen as the contribution of a friction phenomenon since from the Cauchy Schwartz inequality we have

$$
h^{2} u^{2}=\left(\int_{a}^{a+h} \tilde{u}(x, z, t) d z\right)^{2} \leq h \int_{a}^{a+h}|\tilde{u}(x, z, t)|^{2} d z
$$

The equations (25), (26) correspond to a hyperbolic system with the characteristics velocities $u_{-}^{+} c$, where $c=\sqrt{g h}$ is called the wave celerity. The rate $\frac{|u|}{c}$ is called the Froude number. For $|u|=c$ we get the critical velocity. A Froude number greater than 1 corresponds to a supercritic mode, also called torrential mode, and a Froude number less than 1 corresponds to a subcritic mode, also called fluvial mode.

As for the scalar case, the bottom profile $a(x)$ will be approached by a function $a_{\Delta x}$ constant on each cell. We get that way a flat bottom in each cell, that is a homogeneous conservative form of (25), (26) in the inside of each cell. This will allow to use the divergence free argument to perform the projections and get easily the Godunov scheme 
with the usual CFL condition, once the Riemann problem is solved on the two sides of the cell.

The Riemann problem is made of the equations (25), (26) and the initial data

$$
(h(x, 0), u(x, 0))=\left\{\begin{array}{l}
\left(h_{d}, u_{d}\right) \text { for } x>0 \\
\left(h_{g}, u_{g}\right) \text { for } x<0
\end{array}\right.
$$

We consider the case of the following bottom profile

$$
a_{\epsilon}(x)=\left\{\begin{array}{c}
a_{g} \quad \text { for } x \leq 0, \\
a_{g}+\frac{x}{\epsilon}\left(a_{d}-a_{g}\right) \text { for } 0 \leq x \leq \epsilon, \\
a_{d} \quad \text { for } x \geq \epsilon .
\end{array}\right.
$$

for a given $\epsilon>0$ detinated to go to zero. In (28) and (29), the data $a_{g}, h_{g}, u_{g}$ and $a_{d}, h_{d}, u_{d}$ are given constants. We shall build the solution $u_{e}$ for any $\epsilon>0$ and take the limit as $\epsilon$ goes to zero. This solution $u_{\epsilon}$ is made of rarefaction or shock waves propagating with the velocities $u_{-}^{+} c$, on the flat bottom areas, that is either for $x<0$ (this wave is denoted $(L)$ ) or for $x>0$ (this wave is denoted $(R)$ ). On the slope, the remaining wave will be a stationary wave, denoted by $(S)$.

We get the form of a rarefaction wave by writing locally $u$ as a function of $h$, that is $u=u(h)$, in the equations (25), (26). We get, in the case of a flat bottom, the linear system

$$
\left(\begin{array}{cc}
1 & h u^{\prime}+u \\
h u^{\prime}+u & u^{2}+2 h u u^{\prime}+g h
\end{array}\right)\left(\begin{array}{l}
h_{t} \\
h_{x}
\end{array}\right)=\left(\begin{array}{l}
0 \\
0
\end{array}\right) .
$$

Since $h$ is expected to be not constant, the matrix is necessarily a singular matrix, which leads to the condition

$$
h u^{2}-g=0
$$

This can be seen as a differential equation whose solutions are the so called Riemann invariants

$$
u_{-}^{+} 2 \sqrt{g h}=\text { constant } .
$$

For a shock wave, we use the Rankine Hugoniot relations and get that any state $\left(h_{0}, u_{0}\right)$ can be linked only to the states $(h, u)$ satisfying the jump condition

$$
u-u_{0}= \pm\left(h-h_{0}\right) \sqrt{g \frac{h+h_{0}}{2 h h_{0}}} .
$$

The occurrence of a shock wave or a rarefaction wave is ruled by the entropy condition. We get that a $(L)$ wave is characterized by

$$
u= \begin{cases}u_{g}-2\left(\sqrt{g h}-\sqrt{g h_{g}}\right) & \text { for } h \leq h_{g}, \\ u_{g}-\left(h-h_{g}\right) \sqrt{g \frac{h+h_{g}}{2 h h_{g}}} & \text { for } h>h_{g} .\end{cases}
$$

A $(R)$ wave is characterized by 


$$
u= \begin{cases}u_{d}+2\left(\sqrt{g h}-\sqrt{g h_{d}}\right) & \text { for } h \leq h_{d}, \\ u_{d}+\left(h-h_{g}\right) \sqrt{g \frac{h+h_{d}}{2 h h_{d}}} & \text { for } h>h_{d} .\end{cases}
$$

It remains to consider the $(S)$ wave. We first look for a regular solution. By writing $u=u(h)$ as before, we get the linear system, where $k_{\epsilon}=-g \frac{a_{d}-a_{g}}{\epsilon}$, for $0<x<\epsilon$,

$$
\left(\begin{array}{cc}
1 & h u^{\prime}+u \\
h u^{\prime}+u & u^{2}+2 h u u^{\prime}+g h
\end{array}\right)\left(\begin{array}{l}
h_{t} \\
h_{x}
\end{array}\right)=\left(\begin{array}{c}
0 \\
h k_{\epsilon}
\end{array}\right) .
$$

instead of (30). The condition (31) cannot be satisfied for $k_{\epsilon} \neq 0$. This means that the usual Riemann invariants play no role here. We introduce a function $\psi(h)$ such that

$$
\psi^{\prime}(h)=h u^{\prime}(h)^{2}-g
$$

and solve (34). We get

$$
\psi^{\prime}(h) h_{x}=k_{\epsilon}, \quad \psi^{\prime}(h) h_{t}=-k_{\epsilon} \frac{d}{d h}(h u(h)) .
$$

Hence $\psi(h)$ has the form

$$
\psi(h)=k_{\epsilon} x+C(t),
$$

and since $\psi_{x t}=\psi_{t x}$, we get that

$$
\frac{d^{2}}{d h^{2}}(h u(h))=0
$$

that is

$$
h u(h)=A h+B,
$$

where $A$ and $B$ are constants. This allows us to have the expression of $\psi(h)$ and of $C(t)$ in (35). We get

$$
\psi(h) \equiv g h+\frac{B^{2}}{2 h^{2}}=k_{\epsilon}(x-A t)+C_{0},
$$

where $C_{0}$ is a constant. This expression means that a regular $(S)$ wave is a solitary wave, and it is a stationary wave only for $A=0$, that is for a constant flux $h u$. In this case $\psi(h)=g h+\frac{u^{2}}{2}$ which reads as a total energy. Since $u=\frac{B}{h}$, we get that $\psi$ is a convex fonction of $h$, going to infinity when $h \longrightarrow 0$ or $h \longrightarrow \infty$, and minimal for

$$
h=h^{*} \equiv \frac{B^{\frac{2}{3}}}{g^{\frac{1}{3}}},
$$

that is for

$$
u=u^{*} \equiv \sqrt{g h^{*}},
$$

ESA IM: Proc., Vol. 6, SePtembre 1998, 75-90 
which is the critical velocity. From $(37)$ we see that a regular stationary $(S)$ wave has these two properties:

- the mode (fluvial or torrential) can never change on the slope,

- a critical velocity can be reached only on the top of the slope,

- the flux is constant, that is $A=0$.

Let us denote by $h_{l}$ the value of $h$ at $x=0$. We have,

$$
B=h_{l} u_{l}
$$

and from (37) for $x=\epsilon$,

$$
\psi(h(\epsilon))=k_{\epsilon} \epsilon+\psi\left(h_{l}\right)=-g\left(a_{d}-a_{g}\right)+\psi\left(h_{l}\right)
$$

We see that the state at $x=\epsilon$ does not depends on $\epsilon$. Let us denote by $\left(h_{r}, u_{r}\right)$ this state and go to the limit as $\epsilon$ goes to zero (which changes nothing in (38)). We get

$$
\begin{gathered}
h_{r} u_{r}=h_{l} u_{l}(=B) \\
\psi\left(h_{r}\right)-\psi\left(h_{l}\right)=-g\left(a_{d}-a_{g}\right)
\end{gathered}
$$

where the states $\left(h_{r}, u_{r}\right)$ and $\left(h_{l}, u_{l}\right)$ are of the same mode. We remark that the influence of the profile of the slope on the flow is limited to the value of the denivelation only.

We consider now the occurrence of a stationary jump on the slope (29), and denote by $h_{2}$ and $h_{1}$ the values of $h$ on the right side and the left side of the shock respectively. From the Rankine Hugoniot conditions we get

$$
\begin{gathered}
h_{2} u_{2}=h_{1} u_{1}(=B) \\
\theta\left(h_{2}\right)=\theta\left(h_{1}\right)
\end{gathered}
$$

where $\theta(h)$ is a function such that $\theta^{\prime}(h)=h \psi^{\prime}(h)$, which gives here

$$
\theta(h)=g \frac{h^{2}}{2}+\frac{B^{2}}{h^{2}} .
$$

We get from (40)

$$
\frac{\psi\left(h_{2}\right)-\psi\left(h_{1}\right)}{h_{2}-h_{1}}<0
$$

The function $\theta(h)$ is convex, with a minimum at the critical point, as for the function $\psi(h)$ for the same flux $B$. Since $h_{2} \neq h_{1}$, we have necessarily that the states $\left(h_{1}, u_{1}\right)$ and $\left(h_{2}, u_{2}\right)$ are of different modes.

For $B>0$, the velocity field is positive and from the entropy condition $h_{1}<h_{2}$. The state $\left(h_{2}, u_{2}\right)$ is of fluvial mode and the state $\left(h_{1}, u_{1}\right)$ is of torrential mode. For $B<0$, the velocity field is negative and from the entropy condition $h_{1}>h_{2}$. The state $\left(h_{2}, u_{2}\right)$ is of torrential mode and the state $\left(h_{1}, u_{1}\right)$ is of fluvial mode. The position of the stationary jump is determined by the initial data and can be modified when $\epsilon$ goes to zero. Hence the position of a stationary jump is unstable on the slope, and we propose to consider only the cases of stationary jumps standing on the edges of the slope. That way, the only stationary jumps to be considered will be $(L)$ or $(R)$ waves, and not a $(S)$ wave. 


\section{The Riemann solver}

The solution of the Riemann problem (25), (26), (28) (29) is the concatenation of $(L)$, $(S)$ and $(R)$ waves and we are concerned with the limit as $\epsilon$ goes to zero. That way, the $(S)$ wave will reduce to a jump obtained as the limit of a stationary wave of (25), (26). We propose to work with the variables $u$ and $c=\sqrt{g h}$ instead of $h$, since the Riemann invariants become linear in this case. The initial data are

$$
(c, u)= \begin{cases}\left(c_{g}, u_{g}\right) & \text { for } x<0 \\ \left(c_{d}, u_{d}\right) & \text { for } x>0\end{cases}
$$

and we set

$$
K_{0}=-g\left(a_{d}-a_{g}\right) .
$$

We denote $G=\left(c_{g}, u_{g}\right)$ and $D=\left(c_{d}, u_{d}\right)$, and we introduce the two curves $(G L)$ and $(R D)$ defined by

$$
\left\{\begin{array}{c}
c=0 \quad \text { for } u>u_{g}+2 c_{g} \\
u=u_{g}-2\left(c-c_{g}\right) \text { for } 0<c<c_{g} \\
u=u_{g}-\left(c-c_{g}\right) \frac{c+c_{g}}{c c_{g}} \sqrt{\frac{c^{2}+c_{g}^{2}}{2}} \text { for } c \geq c_{g}
\end{array}\right.
$$

and

$$
(R D)\left\{\begin{array}{c}
c=0 \quad \text { for } u<u_{d}-2 c_{d} \\
u=u_{d}+2\left(c-c_{d}\right) \text { for } 0<c<c_{d} \\
u=u_{d}-\left(c-c_{d}\right) \frac{c+c_{d}}{c c_{d}} \sqrt{\frac{c^{2}+c_{d}^{2}}{2}} \text { for } c \geq c_{d}
\end{array}\right.
$$

Following $(G L)$ as $u$ goes from $-\infty$ to $+\infty$ makes $c$ to go from $+\infty$ to 0 and following $(R D)$ as $u$ goes from $-\infty$ to $+\infty$ makes $c$ to go from 0 to $+\infty$. These two curves meet in one point $S=\left(c_{*}, u_{*}\right)$ which is unique for $u_{d}-2 c_{d} \leq u_{g}+2 c_{g}$, and $c_{*}=0$ and $u_{*}$ is undetermined in the other case.

We denote by $L=\left(c_{l}, u_{l}\right)$ the value of the solution on the left side of the $(S)$ jump, that is for $x=0^{-}$, and by $R=\left(c_{r}, u_{r}\right)$ the value on the right side, that is for $x=0^{+}$. These two points are linked by two conditions:

$L$ and $R$ belong to a constant flux curve

$$
c^{2} u=g Q_{0} \quad\left(Q_{0} \text { constant flux }\right)
$$

and $Q_{0}$ is fitted by the Bernoulli condition

$$
\psi\left(h_{r}\right)-\psi\left(h_{l}\right)=K_{0} \quad\left(=-g\left(a_{d}-a_{g}\right)\right),
$$

which is a second linkage between $L$ and $R$. The condition (44) also reads

$$
\left(c_{r}^{2}-c_{l}^{2}\right)\left(1-\frac{g Q_{0}^{2}}{2} \frac{c_{r}^{2}+c_{l}^{2}}{c_{l}^{4} c_{r}^{4}}\right)=K_{0} .
$$

ESA IM: Proc., Vol. 6, Septembre 1998, 75-90 
We first consider the case $K_{0}>0$.

We have several cases and we chooze to present these cases according to the position of $S$.

If $S$ corresponds to a torrential mode with $u_{*}>c_{*}$ we have the following Case 1.

Case 1: Either $L=G$ if $u_{g} \geq c_{g}$ ( $G$ is torrential), or $L$ is the critical point $\left(u_{l}=c_{l}\right)$ of $(G L)$ if $u_{g}<c_{g}$. Since $L$ is determined, we can compute $Q_{0}$ from (43), and the position of $R$ is obtained from (44), with $h_{r}<h_{l}$ ( $R$ is torrential). That way, we have built $L$ and $R$, which is enough for a numerical solver. We get the complete solution by solving a flat bottom Riemann problem with $\left(c_{r}, u_{r}\right)$ as the left data and $\left(c_{d}, u_{d}\right)$ as the right data, and taking the restriction to $x>0$ of this solution.

If $S$ corresponds to a fluvial mode, we consider the critical point $M=\left(c_{M}, u_{M}\right)$ of $(G L)$ (that is $\left.c_{M}=u_{M}\right)$, and the point $N=\left(c_{N}, u_{N}\right)$ at the intersection of $(R D)$ and the flux curve passing through $M$, that is

$$
c_{N}^{2} u_{N}=c_{M}^{2} u_{M}
$$

Then we compute

$$
K_{\max }=\psi\left(h_{N}\right)-\Psi\left(h_{M}\right) .
$$

Now, we have two possible cases.

- either $K_{0}>K_{\max }$, and this case is similar to the Case 1 above,

- or $0 \leq K_{0} \leq K_{\max }$, and we have the following

Case 2 : The point $L$ will belong to $(G L)$, and the point $R$ will belong to $(R D)$, both in fluvial mode. For a given value of the flux $Q\left(=\frac{c_{l}^{2} u_{l}}{g}=\frac{c_{r}^{2} u_{r}}{g}\right)$, we can compute the coordinates of $L$ and $R$, and we can compute the quantity $F(Q)=\psi\left(h_{r}\right)-\psi\left(h_{l}\right)$, which corresponds to a functiont of $Q$. Thus the problems reduces to a single equation of the form

$$
F(Q)=K_{0},
$$

which can be solved by using the dichotomy process for example.

We may remark that for $u_{*}<0$, with a negative flux, the Case 2 still works. When the null flux is reached, we get a flux with $h+a=$ Constant, $u=0$, from $L$ to $R$ for $h_{l}>0$. For $h_{l}=0$, there is a wall reflexion on the right side of the discontinuity, and there is no flux.

If $S$ corresponds to a torrential mode with $u_{*}<-c_{*}$, we consider the critical point $N^{\prime}=\left(c_{N^{\prime}}, u_{N^{\prime}}\right)$ on $(R D)$ and the point $M^{\prime}=\left(c_{M^{\prime}}, u_{M^{\prime}}\right)$ on $(G L)$ belonging to the same flux curve

$$
c_{N^{\prime}}^{2} u_{N^{\prime}}=c_{M^{\prime}}^{2} u_{M^{\prime}}
$$

Then we compute

$$
K_{\text {min }}=\psi\left(h_{N^{\prime}}\right)-\psi\left(h_{M^{\prime}}\right) .
$$


Now we have three possible cases:

- either $K_{0}>K_{\max }$ and this case is to be solved as above in Case 1,

- or $K_{\min } \leq K_{0} \leq K_{\max }$ and this case is similar to the Case 2 above,

- or $K_{0}<K_{\min }$ and we compute $L$ and $R$ as follows.

Case 3: If $G$ is torrential, $L$ and $R$ are on the same flux curve that is $c_{l}^{2} u_{l}=c_{r}^{2} u_{r}$, $L$ belongs to the critical line $u_{l}+c_{l}=0$ and $R$ belongs to $(R D)$, such that $\psi\left(h_{r}\right)=\psi\left(h_{l}\right)+$ $K_{0}, h_{r}>h_{l}$. This characterizes $L$ and $R$, and is sufficient for a numerical computation. The complete Riemann problem is solved by linking $L$ to $G$ by using the solution of a usual flat bottom Riemann problem. If $G$ is fluvial, $L$ is the critical point on $(G L)$ and we proceed as before to obtain $R$.

If $S$ corresponds to a value $c_{*}=0$, then the equation (43) is trivialy satisfied for any $K_{0}$, and (44) is undetermined. The solution is the same as for a flat bottom case since a dry ground appears near the discontinuity of the topography. When the dry ground appears only on one side of the discontinuity, we have a wall reflexion.

For $K_{0}<0$, we get a similar solver, which is the same as above for an observer standing on the opposite bank of the river. We only have to use the new variables

$$
\tilde{u}_{g}=-u_{d}, \tilde{u}_{d}=-u_{g}, \tilde{h}_{g}=h_{d}, \tilde{h}_{d}=h_{g}, \tilde{K}_{0}=-K_{0} .
$$

instead of $u_{g}, u_{d}, h_{g}, h_{d}, K_{0}$, in the solver described above.

\section{A linearized Riemann solver}

The Riemann solver described above works in all cases and is rather difficult to handle. A first linearized solver can be obtained by using the Roe technique, with the variables $m=h u$ and $h$. Such a solver may bring some values near the $m$-axis, which means a small $h$ and consequently a large velocity $u$. From the $C F L$ condition, the time step will severely fall down. The previous analysis clearly shows that a linearization using $c$ and $u$ may be a better choice (see also [2] for the flat bottom case).

We propose here an iterative method using the variables $c$ and $u$, where each step is a linear process. For two states $\left(c_{1}, u_{1}\right)$ and $\left(c_{2}, u_{2}\right)$, we set

$$
\begin{gathered}
\Delta u=u_{2}-u_{1}, \quad \Delta c=c_{2}-c_{1}, \\
\bar{u}=\frac{1}{2}\left(u_{1}+u_{2}\right), \quad \bar{c}=\frac{1}{2}\left(c_{1}+c_{2}\right), \\
\bar{h}=\frac{c_{1}^{2}+c_{2}^{2}}{2 g} .
\end{gathered}
$$

We have

$$
c_{2}^{2} u_{2}-c_{1}^{2} u_{1}=2 \overline{u c} \Delta c+g \bar{h} \Delta u
$$

and

$$
g h_{2}+\frac{u_{2}^{2}}{2}-g h_{1}-\frac{u_{1}^{2}}{2}=2 \bar{c} \Delta c+\bar{u} \Delta u
$$

ESA IM: Proc., Vol. 6, Septembre 1998, 75-90 
We start with two states $\left(c_{1}, u_{1}\right)$ and $\left(c_{2}, u_{2}\right)$, which can be $\left(c_{g}, u_{g}\right)$ and $\left(c_{d}, u_{d}\right)$, and we compute $\bar{c}, \bar{u}$, and $\bar{h}$ as above. Then we iterate the following steps, until convergence:

-1- compute $\Delta c$ and $\Delta u$ solution of the linear system

$$
\begin{gathered}
2 \overline{c u} \Delta c+g \bar{h} \Delta u=0 \\
2 \bar{c} \Delta c+\bar{u} \Delta u=K_{0}
\end{gathered}
$$

-2- compute $c_{1}, u_{1}, c_{2}, u_{2}$ solution of the linear system

$$
\begin{gathered}
u_{1}+2 c_{1}=u_{g}+2 c_{g} \\
u_{2}-2 c_{2}=u_{d}-2 c_{d} \\
c_{2}-c_{1}=\Delta c \\
u_{2}-u_{1}=\Delta u
\end{gathered}
$$

-3- either restart with $c_{1}, u_{1}, c_{2}, u_{2}$, or set $c_{l}=c_{1}, u_{l}=u_{1}, c_{r}=c_{2}, u_{r}=u_{2}$, and stop.

To solve (49) we need to have $\bar{u}^{2} \neq g \bar{h}$ (at least for $K_{0} \neq 0$ ) and (50) always has a solution. In practice, no more than 3 or 4 iterations are needed. And often only one is enough, which corresponds to a really linearized solver.

This solver works on a very wide class of cases, but it is only an approximation, and the error may become large when a strong shock occurs. The property of positiveness of $c_{1}, c_{2}$ may be lost too.

\section{Conclusion}

The Riemann solver including the source term allows to use the Godunov scheme or any finite volume scheme with a CFL condition which does not depend on the source term. It allows to work in a very wide class of values for the variables, even in the linearized version. It can be used in any dimension, since we use it to compute a flux through an interface, which reduces to a one dimension problem. This method has been used for the 2 dimension simulation of a dam break on a random ground, and is particularly efficient near the obstacles or when recovering a dry ground, or also when a dyr ground appears after the flooding. This solver may be extended in order to include non linear friction terms (see [5]). Previous versions of this solver may be found in [9] or [1], with many numerical tests.

\section{References}

[1] C. Bon. Modélisation et simulations numériques d'écoulement hydrauliques et de ruissellement en topographie quelconque. Thèse de doctorat, Bordeaux, 3/7/1997.

[2] Th. Buffard, Th. Gallouet, J.M. Herard. Un schéma simple pour les équations de Saint Venant. C. R. Acad. Sci. Paris, Sér. 1, 326, 1998, 385-390. 
[3] P. Cargo, A.Y. LeRoux. Un schéma équilibre adapté aux modèles d'atmosphère avec terme source. C. R. Acad. Sci. Paris, Sér. 1, 318, 1995, 73-76.

[4] P. Cargo. Nouveaux modèles numériques pour l'hydrodynamique atmosphérique et la Magnétohydrodynamique idéale. Thèse de doctorat, Bordeaux, 11/12/1995.

[5] A. Chinnayya, G. Godinaud, A.Y. LeRoux. A general Riemann solver for Shallow Water equations, with friction and topography terms. To appear.

[6] L. Gosse, A.Y. LeRoux Un schéma équilibre adapté aux lois de conservation scalaires non homogènes. C. R. Acad. Sci. Paris, Sér. 1, 323, 1996, 543-546.

[7] J.M. Greenberg, A.Y. LeRoux. A well balanced scheme for the numerical processing of source terms in hyperbolic equations SIAM Journal on Numerical Analysis, 33-1, 1996, 1-16.

[8] J.M. Greenberg, A.Y. LeRoux,R. Baraille, A. Noussair. Analysis and Approximation of Conservation Laws with Source Terms SIAM Journal on Numerical Analysis, 34-5, 1997, 1980-2007.

[9] O. LeCoz. Un schéma équilibre pour les écoulements à surface libre instationnaires avec bathymétrie. Thèse de doctorat, Bordeaux, 8/7/1996. 